\title{
Percepciones de futuros maestros sobre motricidad en educación infantil: un estudio exploratorio en estudiantes de último curso \\ Perceptions of future teachers on motricity in early childhood education: an exploratory study with last-year students \\ *Josué Prieto Prieto, **David Cerro Herrero \\ *Universidad de Salamanca (España), **Universidad de Extremadura (España)
}

\begin{abstract}
Resumen. La función motora tiene un papel de gran importancia en el desarrollo integral de los individuos, por lo cual la motricidad se presenta como un tema de indudable relevancia dentro de las escuelas. Los estudios previos analizan la importancia de la motricidad en Educación Infantil desde el punto de vista de los docentes, pero consideramos relevante conocer el punto de vista de los futuros docentes para mejorar la formación de los estudiantes universitarios. El presente estudio exploratorio es de tipo descriptivo transversal y de enfoque cuantitativo mediante uso de encuesta, que tiene la intención de conocer las percepciones que los estudiantes del Grado de Maestro en Educación Infantil en relación con la situación de la práctica motora en las aulas. Específicamente, se abordan aspectos relacionados con su percepción hacia su competencia inicial sobre motricidad. Para ello, tuvimos una muestra de 36 estudiantes matriculados en la asignatura de Educación Corporal y Motricidad de cuarto curso del Grado en Educación Infantil en la Facultad de Formación del Profesorado de la Universidad de Extremadura (Cáceres). Todos eran mujeres, con edades comprendidas entre 21 y 25 años. Para medir las percepciones de los estudiantes, utilizamos el Cuestionario para el Análisis de Motricidad en la Educación Infantil (Moreno, et al., 2004). Los resultados nos permiten concluir que los estudiantes perciben la importancia de trabajar la motricidad en las aulas, pero la mayoría de ellos indican que el trabajo realizado no es suficiente. Además, se observó que la competencia para la práctica motriz en los futuros maestros presenta algunas deficiencias. En vista de los resultados, en la educación superior, parece interesante orientar las estrategias de enseñanza y aprendizaje para mejorar la formación de los estudiantes universitarios.
\end{abstract}

Palabras claves: Educación Infantil, Motricidad, Percepciones, Estudiantes universitarios.

Abstract. Conscious of the role of motor function in the integral development of individuals, motricity is presented as a topic of undoubted relevance within schools. Previous studies analyse the importance of motricity in early childhood schools from a point of view of the teachers, but we consider relevant to know the viewpoint of the future teachers in order to enhance the training of college students. The present exploratory study is of a descriptive cross-sectional type with a quantitative approach, using a survey intended to assess the perceptions of students in Early Childhood Education Teacher in relation to the situation of motor practice in the classrooms. Specifically, aspects related to their perception towards their initial training on motricity are addressed. A sample of 36 students enrolled in the subject of Corporal Education and Motricity from the 4th Grade in Early Chilhood Education Teacher at the Faculty of Teacher Training of the University of Extremadura (Cáceres) was selected. All of them were women, aged between 21 and 25 years. To measure students' perceptions, we used the Questionnaire for the Analisys of Motricity in Early Chilhood Education (Moreno, et al., 2004). Results allow us to conclude that students perceive that it is important to work motricity in the classrooms, but most of them indicate that work put into it is not enough. Furthermore, it was observed that the competence on motor practice in future teachers presents some shortcomings. In view of the results, in higher education, it seems interesting to guide teaching and learning strategies towards enhancing the training of college students.

Key words: Early childhood education, Motricity, Perceptions, College students.

\section{Introducción}

Existe una gran variedad de términos para referirnos a la intervención motriz que tiene lugar en las aulas educativas en Educación Infantil (EI), como los de educación física, educación psicomotriz, motricidad, expresión corporal, entre otros (Gil-Madrona, Contreras-Jordaìn, Gonzailez-Viilora \& GoìmezBarreto, 2008). Todos ellos giran en torno a dos conceptos fundamentales, la motricidad y la corporalidad. Según Le Boulch (1981), la motricidad es entendida como la capacidad de un cuerpo para moverse o producir movimiento, y la corporalidad hace referencia al conocimiento inmediato de nuestro cuerpo, sea en estado de reposo o en movimiento, en función de la interrelación de sus partes y de su relación en el espacio y los objetos que nos rodean.

En lo referido al ámbito educativo, Picq y Vayer (1969) consideran la motricidad como una acción pedagógica y psicológica que utiliza los medios de la educación física con el fin de normalizar y mejorar el comportamiento del niño. De

Fecha recepción: 30-03-20. Fecha de aceptación: 29-05-20

Josué Prieto Prieto

josueprieto@usal.es forma concreta, Pons y Arufe (2016), entienden la motricidad como todo trabajo dedicado a la interacción establecida entre el cuerpo, el movimiento, el conocimiento y la emoción, mediante el juego de expresión corporal, motores, de ritmo, de estructuración espacial, etc., y que resulta de gran importancia para el desarrollo del individuo, favoreciendo su capacidad de expresarse y de relacionarse en diferentes contextos sociales. La motricidad infantil es el elemento básico de aprendizaje de la infancia, junto con el juego que es su vehículo didáctico (LaTorre \& López Sánchez, 2009). La educación psicomotriz forma parte de un modelo educativo, que hace años se titula como tratamiento pedagógico del ámbito corporal (Vaca, 1988).

Es bien sabido, que la motricidad es una actividad fundamental para el desarrollo global del niño, ya que éste, en la etapa infantil, está en continuo movimiento. Las sucesivas adquisiciones que el niño realiza en el ámbito motor contribuyen al desarrollo de las capacidades cognitivas, lingüísticas, afectivas, y de inserción social (Espinosa \& Vidanes, 1991; Pacheco, 2015; Piaget, 1969). El desarrollar el área motriz en la etapa infantil contribuye al conocimiento del propio cuerpo, conocer algunas medidas de higiene, alimentación y de salud física además de las principales reglas 
de algunos juegos, normas de educación vial y un sinfín de curiosidades y conceptos que benefician al desarrollo integral del niño (Ruíz, et al., 2003). En la misma línea Gil-Espinosa, Romanace y Nielsen (2018) afirman que el juego y actividad física son dos variables fundamentales para lograr una metodología globalizadora, significativa y motivante que aporte calidad al proceso formativo del alumnado en la etapa de educación infantil.

Seguìn Hernaìndez, Cortegaza y Labrada (2012), el desarrollo motor en el niño se desarrolla mediante actividades propias de la conducta motriz como: movimientos naturales, actitudes perceptivas, actitudes físicas, habilidades motoras, comunicación no verbal y actividades sociales. La práctica psicomotriz permite desarrollar las distintas capacidades infantiles a partir de la interacción del cuerpo con el entorno $y$, en concreto, persigue los siguientes fines: a) el conocimiento, la comprensión y el dominio de sí mismo; b) el conocimiento y la comprensión del otro; c) el conocimiento y la comprensión de entorno y d) la comprensión de las relaciones entre uno mismo, los demás y el entorno (Sugrañes \& Ángel, citado en Mérida-Serrano, Olivares-García \& GonzálezAlfaya, 2018)

Por todo lo anterior, es evidente la importancia de ésta y de su obligada presencia en el contexto escolar, en el que cumple una relevante misión educadora en todas sus facetas, tanto en el desarrollo físico y motriz como en el desarrollo mental (Pacheco, 2015). La Ley Orgánica 2/2006, de 3 de mayo, de Educación (LOE), es el documento legal que regula en la actualidad la etapa de EI. Según esta, la finalidad de la etapa es «contribuir al desarrollo físico, afectivo, social e intelectual de los niños y niñas». Según la LOE (2006), la etapa se estructura u organiza en tres áreas, con un carácter global e integrador, estableciéndose objetivos y contenidos por cada una de ellas, formulados en base a capacidades cognitivas, motrices, afectivas, de relación interpersonal y de inserción social (Arac'ilgar \& Cihan, 2018). El tratamiento de la motricidad en el contexto escolar, queda justificado, por su presencia de forma explícita e implícita en el currículo educativo y su contribución al desarrollo de todas las capacidades que establece el mismo para la etapa infantil (Camina-López, 2013).

Pero además de la prescripción curricular, la importancia de la motricidad en el contexto escolar, queda también patente en las percepciones de los docentes, en cuanto a la incuestionable importancia que le otorgan a ésta los maestros de la etapa infantil, y su relación con el desarrollo en las áreas cognitiva, social y emocional (Álvarez \& Couto, 2020; Díaz \& Sosa, 2016; Latorre 2007; Moreno, López, Gutiérrez, Cascada, \& Fernández, 2004). Sin embargo, resulta paradójico que, a pesar de la importancia dada por los docentes, los estudios previos evidencian un escaso número de horas dedicados a la motricidad o educación física en las aulas de infantil (Da Fonseca, Garrote, Todoli \& Zenarruzabeitia, 2014; Moreno et al., 2004; Quirós, 2000; Valdemoros, Sanz, Ponce de León, \& Alonso Ruíz, 2018). La motricidad no se encuentra en el nivel deseado en el sistema educativo preescolar según los propios docentes, por diversas razones entre las que destacan que, no está lo suficientemente incluida en el programa educativo (Sugranbes-Justafreì \& Aìngel-Ferrer, 2007), no tienen un marco curricular adecuado en nuestro ordenamiento profesional universitario (Aguado-Ruiìz, 2014; Dopico-Pedre, 2016; Gambau, 2015; Quiroìs, 2000), falta de formación específica de los docentes (Solís, Prieto, Nistal \& Vázquez, 2017; Pons \& Arufe, 2016), o carece de infraestructura y herramientas didácticas necesarias (Diaz \& Sosa, 2006; Fraile-Garciìa,Ibaibarriaga-Toset \& Mon-Loìpez, 2019; SaìenzLoìpez 1999; Shingjergji, 2013).

Como evidencian los precitados estudios, la situación de la motricidad en el contexto escolar, ha sido valorada desde la opinión de los maestros en activo en las aulas de EI, siendo escasos los estudios acerca de la percepción de los fututos docentes durante el periodo de formación inicial en las universidades. En este sentido, Marinšek, Jurak y Kovaè, (2020) encontraron diferencias en la percepción de su competencia personal para la docencia de la educación física en EI entre docentes en activo y futuros docentes. Convenimos que la opinión acerca de la transcendencia de la motricidad escolar, comienza a forjarse en las aulas universitarias, donde sabemos que la formación en relación a esta, es escasa en contenidos y presenta carencias, como manifiestan estudios previos (Rodríguez \& Hernández, 2018; Alonso \& Pazos, 2020; González, Sendón, Díaz, \& Gómez, 2018). En concreto en relación a la didáctica de la motricidad infantil en futuros docentes, Martínez-Bello y Bernabé-Villodre, (2019) ponen de manifiesto que la relación de estos con las aulas es escasa al limitarse al Prácticum. Dentro de la formación universitaria, el Prácticum es una pieza clave en la adquisición de competencias (Muradás \& Porta, 2007) pero también en aspectos interpersonales relacionados con la construcción de una visión personal y crítica de la realidad educativa (Mendoza \& Cobarrubias, 2014). Consideramos por ello que la formación inicial, y en particular el periodo de prácticas, puede ejercer una influencia en la percepción de los futuros docentes de EI. En el caso concreto de la Educación Física, la literatura previa destaca que la percepción de los futuros docentes se halla condicionada por el rol marginal propio de esta y de sus experiencias previas (Gonzalez-Calvo \& Martínez-Álvares, 2018; Torre, 2012).

Por todo ello, el objetivo de este estudio se centra en conocer la percepción acerca de la motricidad de los estudiantes que se encuentran cerca de concluir sus estudios de Grado de Maestro en EI, así como analizar la influencia en dicha percepción de variables relacionadas con el centro, el tutor o la propia práctica.

\section{Material y método}

\section{Método}

Se plantea un estudio exploratorio con el propósito de examinar un tema poco estudiado y contribuir a la escasas bibliografia existente (Hernández, Fernández \& Baptista, 2003, p.115), que emplea un diseño de tipo descriptivo transversal con datos de carácter cuantitativo mediante el uso de encuesta.

\section{Participantes}

Se uso una muestra no probabilística por conveniencia. Los participantes del estudio fueron estudiantes de cuarto curso del Grado en Maestro en EI de la Facultad de Formación del Profesorado de la Universidad de Extremadura 
(Cáceres), matriculados en la asignatura El Cuerpo y la Motricidad en del Itinerario de Educación Especial en EI, y que se encontraban cursando el periodo de prácticas docentes (Prácticum) La muestra está constituida por la totalidad de estudiantes matriculados en la asignatura, 36 mujeres de edad comprendidas entre 21 y 25 años $(\mathrm{M}=22.08$; $\mathrm{dt}=0.91)$. Asimismo, tomaron parte del estudio los 36 maestros/as que tutorizaron dichas prácticas en los centros educativos (tabla 1) Del total de maestros-tutores, 32 (88,9\%) eran mujeres y 4 (11.1\%) hombres, con una edad media de 41.58 años ( $d t=9.92)$, siendo el rango de 23 a 60 años. La mayor parte tiene titulación de maestra en EI (91.7\%), desempeña su puesto docente en un centro educativo público (69.4\%), y acumula más de 10 años de experiencia (66.7\%). Respecto a las variables relacionadas con la motricidad, imparten principalmente en segundo ciclo (88.8\%), los espacios utilizados principalmente son la sala de psicomotricidad (36.1\%), el aula ordinaria (27.8\%) o el pabellón (22.2\%), la dedicación semanal es de 1 hora o menos (44.4\%), el grupo de alumnos es superior a 15 niños, y el momento dedicado a su tratamiento es a última hora de la mañana $(66.7 \%)$. Los principales problemas para trabajar la motricidad reportados por las maestras-tutoras son el número de alumnos (30.6\%), las instalaciones (19.4\%) y la formación (19.4\%).

\begin{tabular}{|c|c|c|c|}
\hline Variables dependientes & & $\mathbf{N}$ & $\%$ \\
\hline \multirow{2}{*}{ Sexo } & Mujer & 32 & 88.9 \\
\hline & Hombre & 4 & 11.1 \\
\hline \multirow{3}{*}{ Tipología de centro } & Público & 25 & 69.4 \\
\hline & Concertado & 10 & 27.8 \\
\hline & Privado & 1 & 2.8 \\
\hline \multirow{2}{*}{ Titulación académica } & Maestro Infantil & 33 & 91.7 \\
\hline & Otra & 3 & 8.3 \\
\hline \multirow{3}{*}{ Años de experiencia } & Menos de 5 años & 9 & 25.0 \\
\hline & Entre 5 y 10 años & 3 & 8.3 \\
\hline & Más de 10 años & 24 & 66.7 \\
\hline \multirow{5}{*}{ Edad sobre la que imparte } & 1-2 años & 2 & 5.6 \\
\hline & $2-3$ años & 2 & 5.6 \\
\hline & 3-4 años & 17 & 47.2 \\
\hline & 4-5 años & 7 & 19.4 \\
\hline & 5-6 años & 8 & 22.2 \\
\hline \multirow{5}{*}{ Espacio motricidad } & Aula ordinaria & 10 & 27.8 \\
\hline & Patio escolar & 3 & 8.3 \\
\hline & Pabellón & 8 & 22.2 \\
\hline & Sala psicomotricidad & 13 & 36.1 \\
\hline & Otra & 2 & 5.6 \\
\hline \multirow{3}{*}{$\begin{array}{l}\text { Tiempo semanal dedicado a la } \\
\text { motricidad }\end{array}$} & $1 / 2-1$ hora & 16 & 44.4 \\
\hline & $11 / 2-2$ hora & 13 & 36.1 \\
\hline & 3 horas o más & 7 & 19.5 \\
\hline \multirow{2}{*}{ Número de alumnos } & Menos de 15 & 2 & 5.6 \\
\hline & Más de 15 & 34 & 94.4 \\
\hline \multirow{2}{*}{ Momento de la motricidad } & A primera hora & 12 & 33.3 \\
\hline & A última hora & 24 & 66.7 \\
\hline \multirow{5}{*}{$\begin{array}{l}\text { Problemas para trabajar la } \\
\text { motricidad }\end{array}$} & Instalaciones & 7 & 19.4 \\
\hline & Materiales & 4 & 11.1 \\
\hline & $\mathrm{N}^{\circ}$ alumnos & 11 & 30.6 \\
\hline & Formación & 7 & 19.4 \\
\hline & Otros & 7 & 19.4 \\
\hline
\end{tabular}

\section{Instrumento}

Para la recogida de la información se utilizó el Cuestionario para el Análisis de la Motricidad en EI (C.A.M.E.I.) (Moreno, et al, 2004), estructurado en dos partes. La primera, que fue respondida por los maestros-tutores, agrupa 12 ítems de carácter socio-académico en relación al entorno docente y a las condiciones del centro-aula, y que permite obtener una descripción del perfil del tutor de prácticas, y poder conocer su influencia sobre otras variables del estudio. La segunda, que fue respondida por los estudiantes, está compuesta de 39 ítems, que recoge información relacionada con el currículum, el interés y actitud del profesorado, la metodología y la formación docente. Está a su vez dividida en dos apartados; el primero con 27 ítems responden a una escala tipo Likert de 1 a 4, donde 1 es «nada de acuerdo «, y 4 es «totalmente de acuerdo» y que valoran el grado de satisfacción respecto a cada uno de los ítems. El segundo apartado agrupa 12 ítems con respuesta dicotómica «si» o «no». Los ítems 12, 17, 18, 25 y 27 deben ser invertidos para su análisis puesto que estas preguntas son presentadas de forma negativa. El índice de fiabilidad del instrumento original a través de la prueba Alpha de Cronbach muestra una alta consistencia interna; siendo para los primero 27 ítems de á=.87 mientras que para los segundos 12 ítems fue de á=.81. En el presente estudio la fiabilidad de la escala mediante alfa de Cronbach arrojó valores excelentes para la primera sección de la escala (á=.91), mientras que en la segunda sección mostró una baja consistencia interna $(\dot{a}=.63)$ (Streiner, 2003).

\section{Recogida de datos}

Los estudiantes y maestros-tutores fueron informados del propósito del estudio y recibieron las instrucciones previas del cuestionario empleado. Se adviertió de la importancia de la sinceridad y se remarcó que el carácter totalmente anónimo del cuestionario. Todos dieron su consentimiento informado por escrito. Los estudiantes recopilaron mediante entrevista cara a cara con sus maestros-tutores las respuestas relativas a las características socio-académicas de la primera parte del cuestionario. Con posterioridad los estudiantes contestaron a los ítems correspondientes a la segunda parte del instrumento desde su perspectiva de futuros docentes y bajo la experiencia de la formación inicial y tras los dos periodos de prácticas docentes que realizan en el grado. Esta segunda parte se desarrolló en una tutoría grupal bajo la presencia de los investigadores, el tiempo medio de respuesta para este apartado del cuestionario fue de $10 \mathrm{minu}-$ tos. La identidad de los estudiantes, maestros-tutores, y centros escolares ha sido mantenida en el anonimato para asegurar su participación y confidencialidad.

\section{Análisis estadístico}

En primer lugar, se realizó un análisis descriptivo de las variables del estudio para obtener el perfil socio-académico de los maestros-tutores, a través de medias y desviaciones típicas en las variables continuas y, en el caso de variables categóricas, a través de frecuencias y porcentajes. Los descriptivos de media, mediana desviación típica y percentiles ( 25 y 75) fueron calculados para los ítems de la primera sección (1-27), y la distribución de frecuencias y porcentajes para los ítems de la segunda sección (28-39). El análisis de la fiabilidad del instrumento fue realizado mediante el cálculo del coeficiente de á de Cronbach, mientras que el análisis factorial exploratorio con el método de verosimilitud fue empleado para analizar la validez de constructo, previa realización del test de esfericidad de Bartlett de adecuación muestral de Kaiser-Meyer-Olkin.

La naturaleza de los datos recomendó recurrir a pruebas no paramétricas. El análisis de correlaciones mediante Rho de Spearman para muestras no paramétricas fue realizado para determinar la asociación entre las variables. La prueba de y Chi-cuadrado de Pearson $(\div 2)$ fue utilizada para explorar la distribución de frecuencias entre las variables categóricas del estudio. Todos los análisis fueron realizados utilizando el paquete estadístico SPSS versión 23.0 para Windows (IBM, Armonk, Nueva York). La significación estadística fue $p<.05$. 


\section{Resultados}

En la tabla 2 se muestran las puntuaciones medias y desviaciones en los ítems correspondientes a la primera sección del cuestionario, desde la perspectiva de los futuros docentes. Los ítems más valorados por las estudiantes fueron la importancia de la formación permanente de los docentes $(\mathrm{M}=3.83 ; \mathrm{dt}=.964)$, de la utilización del juego para el desarrollo de la motricidad ( $\mathrm{M}=3.78 ; \mathrm{dt}=.422$ ), y la necesidad de establecer un clima de confianza y espontaneidad $(\mathrm{M}=3.78$; $\mathrm{dt}=.485)$. Además, atendiendo a las puntuaciones tras la corrección de las preguntas invertidas, se incluye en este grupo la relación de la motricidad con las áreas cognitivas, social y emocional $(\mathrm{M}=3.78 ; \mathrm{dt}=.722)$. Por el contrario, los ítems menos valorados son la falta de contenidos tratados en la titulación ( $\mathrm{M}=2.36$; $\mathrm{dt}=.961)$, el bajo predominio de aprendizaje a través de la búsqueda $(\mathrm{M}=2.39 ; \mathrm{dt}=.964)$, y no facilitar soluciones a los problemas motrices $(\mathrm{M}=2.50 ; \mathrm{dt}=1.056)$.

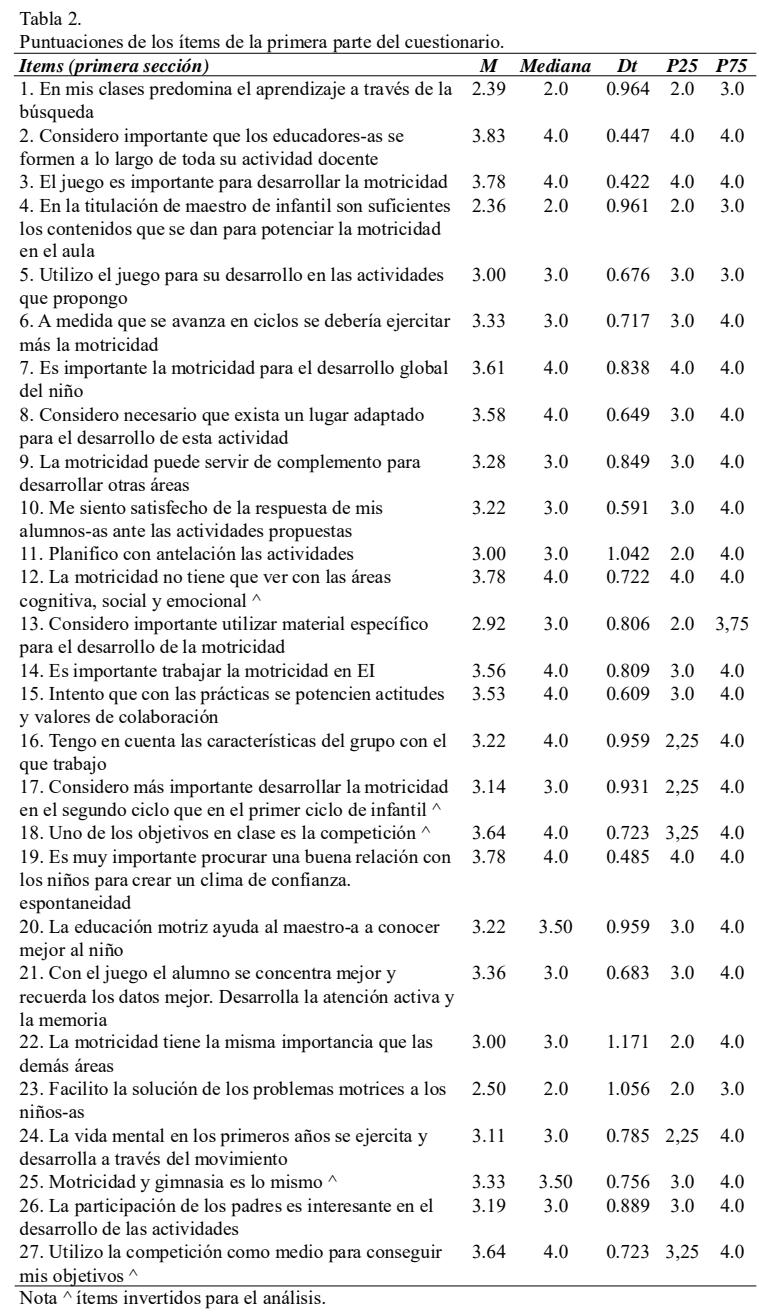

En la tabla 3 se muestran la distribución de frecuencias y porcentajes correspondiente a los ítems de la segunda sección del cuestionario, con respuesta dicotómica «si»o «no». Casi la totalidad de estudiantes $(94.4 \%)$ consideran importante el desarrollo del área motriz, y un alto porcentaje de estudiantes le gusta trabajar la motricidad $(72.2 \%)$ y se siente capacitado para desarrollar la motricidad (61.1\%). Respecto al trabajo de la motricidad, el estudiantado considera que no es suficientemente trabajada en las aulas (69.4\%), y des- taca un $41.7 \%$ de estudiantes que consideran debe ser impartida por el profesor especialista de Educación Física. También, manifiestan que la planificación de la materia no es conjunta en un mismo nivel (61.1\%), y no hay una interrelación con los niveles superiores (61.1\%). Asimismo, expresan que las sesiones de motricidad se realizan con todos los alumnos (66.7\%), y no todos utilizan ropas adecuadas (66.7\%).

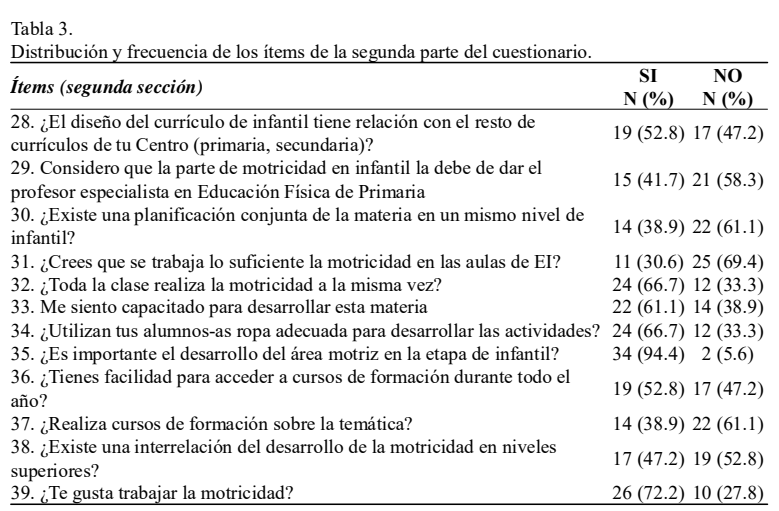

La primera sección del instrumento fue sometida al análisis factorial exploratorio (AFE) por el método de componentes principales mediante rotación varimax con normalización de Kaiser, con el propósito de extraer dimensiones o factores que explican las respuestas a los ítems. El índice KaiserMeyer-Olkin confirmó la validez del análisis $(\mathrm{KMO}=.54)$, considerado como apropiado (Field, 2009). El test de esfericidad de Bartlett $\left(\div{ }_{325}{ }^{2}=647.926 ; \mathrm{p}<.001\right)$, indica que las correlaciones entre los ítems son suficientes para el análisis factorial. El AFE de los 27 ítems resultó una estructura dimensional formada por 6 dimensiones denominadas que arrojan de forma conjunta un $74.82 \%$ de la varianza total explicada. La estructura factorial no resultó clara, debido al bajo número de sujetos de la muestra y el elevado número de variables (ítems) de la escala. Algunos factores no pueden ser considerados subyacentes ya que no agrupan un número suficiente de ítems, o algunos de los ítems pueden explicar varios factores según sus puntuaciones $(>.040)$. Los factores extraídos orientan a la determinación de dimensiones relacionadas con la importancia percibida $(20,21,9,12,14,24$, 22), la competencia pedagógica $(5,23,15,25,11,16,27,18,17$, 10) o las necesidades para una práctica adecuada $(8,13)$.

Respecto al grado de asociación de las variables socioacadémicas con los ítems del cuestionario, la matriz de correlaciones reportó asociaciones a nivel de significación $p<.05$ y $p<.01$ (tabla 4). No se encontraron asociaciones entre la variable edad del maestro-tutor con ninguno de los ítems del cuestionario. En cambio si para la variable de años de experiencia del maestro-tutor, que se asocia de forma negativa (a mayor experiencia menor nivel de acuerdo en el ítem) con el predominio de una metodología de aprendizaje a través de la búsqueda (rho=-.450; $\mathrm{p}=.006)$ y la capacidad de facilitar soluciones a los problemas motrices de los niños $(r h o=-352 ; p=.035)$. Y de forma positiva con el sentimiento de capacitación para desarrollar la materia $(\mathrm{rho}=.337 \mathrm{p}=.044), \mathrm{y}$ el gusto por trabajar la motricidad ( $r h o=.335 ; \mathrm{p}=.046)$. Respecto a la titulación del maestro-tutor se asocia de forma positiva con la realización de la motricidad en gran grupo, siendo el nivel de acuerdo con el ítem mayor en los técnicos $\operatorname{de} \mathrm{EI}(\mathrm{rho}=.426 ; \mathrm{p}=.010$ 
Cabe señalar en cuanto a la variable tipología de centro (público-privado), esta se asocia de forma negativa (menor acuerdo con el ítem en centros privados), con los ítems relacionados con la competición, como es la inclusión de esta como uno de los objetivos de la clase $(\mathrm{r}=-372 ; \mathrm{p}=.025)$ y la utilización como medio para conseguir los objetivos ( $\mathrm{r}=-372$; $\mathrm{p}=.025$ ). Además, con la existencia de una planificación conjunta de la materia en un mismo nivel de infantil ( $\mathrm{r}=-.349$; $\mathrm{p}=.037)$ y el uso de ropa adecuada por los alumnos (rho=$.466 ; p=.004)$. En cambio, la asociación es positiva. con el interés en la participación de los padres ( $\mathrm{r}=.339 ; \mathrm{p}=.043)$.

La variable espacio empleado para la motricidad, reportó una asociación positiva con una mayor importancia otorgada a la motricidad en el segundo nivel ( $r$ ho $=.338 ; \mathrm{p}=.044)$, con la participación de los padres $(r h o=.372 ; p=.026)$ y el pensamiento de que debe ser impartica por un especialista de $E F(r h o=.421 ; p=.011)$. En cambio, se asocia de forma negativa con el uso de ropa adecuada ( $r h o=-.375 ; \mathrm{p}=.024)$. La variable tiempo dedicado a la motricidad se asocia de for-

\begin{tabular}{|c|c|c|c|c|c|c|}
\hline Ítems & Edad & Exper. & Titulo & Tipo & Espacio & Tiempo \\
\hline P1 &,- 232 &,$- 450^{* *}$ &,- 137 &,- 181 & ,137 &, $342^{*}$ \\
\hline P2 &, 056 &,- 103 & ,121 & ,101 &,- 005 &,- 046 \\
\hline P3 & ,097 & ,035 & ,161 &,- 068 &, 111 & ,083 \\
\hline P4 &, 072 & ,056 & 0,000 &, 250 &,- 131 &,- 102 \\
\hline P5 &,- 026 &,- 123 & ,151 &,- 262 &, 251 & ,224 \\
\hline P6 &,- 178 &,- 071 &, 323 & ,056 &, 316 &,- 070 \\
\hline P7 &,- 180 &,- 277 & ,160 &,- 098 &,- 038 & ,086 \\
\hline P8 &,- 101 &,- 087 & ,017 &,- 215 & 167 &,- 266 \\
\hline P9 &,- 063 &,- 176 &,- 190 &,- 171 & , 157 & ,410* \\
\hline P10 & ,066 &,- 001 &,- 135 &,- 028 & ,138 & ,152 \\
\hline P11 &,- 047 &,- 186 &,- 020 &, 117 & ,087 & ,431 \\
\hline P12 &,- 244 &,- 246 &,- 195 &,- 158 & ,038 & ,225 \\
\hline P13 &,- 104 &,- 114 &,- 238 & 209 & ,008 & -,191 \\
\hline P14 &,- 135 &,- 247 & 0,000 &, 120 & 249 & ,259 \\
\hline P15 &, 152 &,- 059 &,- 022 & , 109 & ,245 & 201 \\
\hline P16 &,- 002 &,- 232 &,- 222 & ,084 & ,016 &, $361^{*}$ \\
\hline P17 &,- 175 &,- 208 &,- 201 &,- 128 &, $338^{*}$ & 188 \\
\hline P18 &,- 094 &,- 043 & ,172 &,$- 372^{*}$ & , 189 & ,024 \\
\hline P19 &,- 092 &,- 187 & ,148 &,- 150 & ,021 & ,174 \\
\hline P20 &,- 116 &,- 076 &,- 021 &,- 090 & ,087 & 244 \\
\hline P21 & ,095 &,- 053 &,- 037 &,- 105 & ,058 & 100 \\
\hline P22 &,- 163 &,- 224 & ,026 &,- 057 & , 156 & ,240 \\
\hline P23 &,- 274 &,$- 352^{*}$ &,- 045 & ,154 &,- 033 & ,289 \\
\hline P24 &,- 046 &,- 306 &,- 062 &,- 100 & ,209 & ,113 \\
\hline P25 &,- 097 &,- 225 & ,032 &, 146 & ,147 &,- 012 \\
\hline P26 &,- 067 &,- 078 & ,010 &, $339^{*}$ &, $372^{*}$ & , 107 \\
\hline P27 &,- 094 &,- 043 & ,172 &,$- 372^{*}$ & , 189 & ,024 \\
\hline P28 &,- 121 &,- 271 & ,117 &,- 120 &,- 092 & ,166 \\
\hline P29 &,- 019 &,- 029 &, 255 & ,084 & ,421 ${ }^{*}$ &,- 006 \\
\hline P30 &,- 008 & ,010 & ,034 &,$- 349^{*}$ &,- 034 &,- 164 \\
\hline P31 &,- 242 &,- 094 &, 200 &,- 069 &,- 064 & ,069 \\
\hline P32 &, 014 & ,041 &, $426^{* *}$ &,- 180 & ,012 & ,211 \\
\hline P33 &, 248 &, $337^{*}$ &,- 034 &,- 287 &,- 180 &,- 079 \\
\hline P34 &, 080 & ,267 & 0,000 &,$- 466^{* *}$ &,$- 375^{*}$ & ,003 \\
\hline P35 &,- 147 &,- 021 &,- 073 &,- 160 &,- 316 &,- 102 \\
\hline P36 &,- 110 &,- 058 & ,117 &,- 120 &,- 198 & ,006 \\
\hline P37 &, 232 &, 307 & ,034 &, 048 &,- 146 &,- 082 \\
\hline P38 &,- 054 &,- 029 & ,084 &, 120 &,- 145 &,$- 357^{*}$ \\
\hline P39 &, 138 &, $335^{*}$ &, 037 &,- 149 &,- 199 &,- 327 \\
\hline
\end{tabular}

ma positiva (a mayor tiempo mayor nivel de acuerdo) con el predominio de una metodología de aprendizaje a través de la búsqueda $(\mathrm{rho}=.342 ; \mathrm{p}=.041)$, con la consideración de servir como complemento para desarrollar tareas $(\mathrm{rho}=.410 ; \mathrm{p}=.013)$, con la planificación de actividades con antelación ( $\mathrm{rho}=.431$; $\mathrm{p}=.009$ ), con tener en cuenta las características del grupo $(\mathrm{rho}=.361 ; \mathrm{p}=.031)$. En cambio se asocia de forma negativa con la consideración de estar interrelacionada con niveles superiores (rho=-.357; $\mathrm{p}=.033$ ).

Por último, las tablas de contigencia permitieron examinar la independiendia en las distribuciones de las variables socio-académicas y los ítems del cuestionario (figura 1). La variable tipología de centros fue recodificada en una variable dicotómica -público y privada (agrupa centros concertados y privados). Los resultados permitieron contrastar diferencias en la distribución de frecuencias en relación a la planificación intranivel e internivel $\left(\div^{2}=4.08 ; p=.043\right)$ o el uso de ropa adecuada $(\div 2=7.92 ; p=.005)$, que han sido mejor valorados por los estudiantes asignados a centros privados-concertados. En relación a la variable años de experiencia del maestro-tutor, los datos reportan diferencias en la distribución de frecuencias en relación al predominio del aprendizaje a través de la búsqueda $\left(\div^{2}=13.71 ; p=.033\right)$, y dar solución a los problemas motrices de niños-as $\left(\div^{2}=24.82 ; p=.000\right)$

\section{Discusión}

El presente estudio exploratorio tenía como objetivo analizar la situación del tratamiento de la motricidad en EI desde la perspectiva de los futuros docentes durante su formación inicial, así como explorar posibles influencias del centro y/o tutor asignado, o de la propia práctica motríz. Pues bien, hasta donde sabemos, no existen estudios previos en la literatura que analicen la percepción de los estudiantes de maestro en infantil durante su formación inicial.

Si bien el propósito de este estudio no es analizar el perfil 
docente de los maestros en la etapa de EI, las características de los tutores participantes en el estudio son acorde a los rasgos propios que muestran los estudios previos (Castro \& García-Ruíz, 2013; García-Esteban \& Murga-Menoyo, 2015; Peña \& Vicente, 2019), siendo mayoritariamente mujeres con titulación de maestro en EI, de edad entre 36-40 años y con experiencia de 10 años o más. Respecto a la edad de los docentes, los estudios precitados reportan que es más elevada en centros urbanos y se incrementan los años de experiencia frente a los docentes de ámbito rural. Esto puede ser debido en parte a la dificultad de conseguir destino en los centros urbanos, habitualmente más solicitados, y que son designados por puntuación en experiencia entre otros criterios.

En la línea de estudios previos, la percepción de los futuros docentes es similar a la de los docentes en activo en relación a la importancia que otorgan a la motricidad en la etapa, considerada por ambos necesaria y de gran importancia para la formación de los niños (Alonso \& Pazos, 2020; Díaz \& Quintana, 2016; Moreno, et al., 2004; Simo \& Espada, 2013). La mayoría de los estudiantes otorgan un alto valor a la inclusión de esta en el currículo, además perciben su relación con los dominios cognitivo, social y emocional, y conciben interdisciplinariedad con otras áreas. Los estudiantes manifiestan interés y gusto por trabajar la motricidad en las aulas, y marcan diferencias con la concepción tradicional de gimnasia, identificando como base de ella el juego y separándola de la competición como objetivo o medio. Estos hechos, nos hace pensar que los egresados universitarios llegarán a las aulas con el convencimiento de la transcendencia que tiene el ámbito motriz y un concepto adecuado del área.

En cambio, tras el acercamiento a la realidad educativa durante las prácticas docentes, los estudiantes toman conciencia de que no se trabaja lo suficiente en las aulas, percepción que es compartida por los propios docentes en activo (Alonso \& Pazos, 2020; Díaz \& Quintana, 2016; Moreno, et al., 2004; Simo \& Espada, 2013), y que argumenta la necesidad de aumentar el número de horas y de darle una mayor presencia en el día a día del aula, Al respecto, sorprende, que los estudiantes a punto de finalizar sus estudios universitarios, consideren que la motricidad debe ser impartida por el especialista de educación física de primaria. Es posible que el estudiante ponga en duda su competencia para trabajar la motricidad en las aulas en su futuro docente, debido a su percepción manifiesta de la escasez de contenidos específicos dedicados a la motricidad en la titulación. Estudios previos como los de Berruezo (2000) y Palomero (2007), fundamentan este hecho, y evidencian que estos contenidos del plan de estudios en esta materia son inferiores en cuanto a número de asignaturas frente a los titulados en Educación Física en Primaria. Abilleira y Fernández (2017) también hallaron en docentes en activo una baja percepción de su nivel de formación en un área concreta de la motricidad, la expresión corporal. Por todo ello son muchos los centros escolares que justifican por la necesidad de que los contenidos de motricidad sean impartidos por los especialistas de educación física de primaria, aun siendo de una etapa diferente. En cambio, Campos, Lasaga, y Ries (2013) consideran que la mayoría de los maestros de EI se sienten capacitados para impartir motricidad, por lo que se puede considerar que la formación inicial que recibían los maestros de su estudio es válida para tal fin.

En relación a las competencias del estudiante, cabe destacar las carencias percibidas en cuanto a la intervención motriz, con un bajo predominio en el manejo de un aprendizaje a través de la búsqueda, una baja satisfacción en las respuestas motrices logradas en los alumnos, la falta de planificación previa de las actividades, ser capaz de contemplar las características del grupo, o la dificultad de dar solución a los problemas motrices. Es evidente que estas carencias son propias de la fase formativa en la que se encuentran y la escasa experiencia que acumulan. No se han encontrado estudios previos sobre la visión de los futuros docentes de EI y su competencia en impartición de contenidos de motricidad. Los resultados del presente estudio pueden ser utilizados para orientar el tratamiento de los contenidos específicos impartidos en la titulación de Grado en EI de cara a desarrollar las competencias en las que más carencias manifiestan los estudiantes. A pesar de las limitaciones en sus competencias pedagógicas, los futuros docentes manifiestan no realizar en este momento de su formación inicial cursos de formación sobre motricidad. En comparación con los datos de docentes en activo, este hecho no está lejos de la realidad, ya que los estudios previos no sitúan a la especialización de los maestros de EI en motricidad en un aspecto prioritario, argumentando que la oferta formativa de escasa y de baja calidad (Díaz \& Sosa, 2016; Quirós, 2000).

En cuanto a las necesidades para el desarrollo de una educación motriz adecuada, disponer de un espacio y unos materiales idóneos para el tratamiento de la motricidad, son un factor de motivación y de estimulación significativos capaces de excitar y dirigir específicamente el comportamiento infantil (Madrona, Jordán \& Barreto, 2008). Estudios previos sitúan a instalaciones y materiales entre las principales limitaciones de los maestros para el trabajo de la motricidad de los centros escolares. En este estudio, los estudiantes otorgan mayor importancia a la disponibilidad de un espacio adecuado, frente al uso de materiales específicos. En relación al espacio, cabría pensar que la sala de psicomotricidad señalada por los maestros de este estudio no corresponda a un aula específica, sino al gimnasio, identificado en los estudios como la instalación prioritaria para la práctica de educación física (Crespo, 2006; Pons \& Arufe, 2016). En cuanto a la menor importancia concebida a los materiales, es propio de esta etapa la elaboración de materiales de autoconstrucción adaptados a las necesidades, y por tanto la prescindibilidad de materiales específicos de educación física.

Por último, no se ha encontrado una asociación clara ni diferencias significativas entre las percepciones de los estudiantes y el perfil del maestro-tutor de prácticas. De forma no concluyente se han obtenido limitadas inferencias. En cuanto a los años de experiencia del maestro-tutor asignado, en aspectos como el predominio de un aprendizaje mediante la búsqueda y la capacidad de facilitar soluciones a los problemas de los niños-as, que han sido mejor valorados por estudiantes asignados a maestros-tutores con menos experiencia. También respecto a la tipología de los centros en los que los estudiantes cursan sus prácticas docentes, con aspectos como la participación de los padres, el uso de ropa adecuada o la planificación intranivel e internivel, que han sido 
más valorados por los estudiantes asignados a centros privados-concertados.

\section{Limitaciones}

Este trabajo se trata de un estudio exploratorio que presenta determinadas limitaciones. La escasa muestra en general, y en particular de determinada tipología de centros, no permitió llevar a cabo un análisis exhaustivo con el propósito de establecer diferencias entre centros públicos y privados, o por niveles de enseñanza, entre otros. Asimismo, el tamaño ha limitado la reducción de la escala a dimensiones mediante el análisis factorial, que hubiera ampliado las posibilidades en el análisis de diferencias con las variables independientes.

\section{Prospectiva de futuro}

En la línea de este trabajo es interesante ampliar la muestra del estudio e incluir mayor número de centros de diferente tipología (colegios rurales agrupados, centros de educación especial, escuelas infantiles, entre otros), con el propósito de realizar un análisis comparativo que permita extraer diferencias entre estos. Asimismo, Es de interés el desarrollo de otro instrumento adecuado para valorar las competencias del docente de educación física en educación infantil. En este sentido un reciente estudio ha validado un el Cuestionario de Desarrollo Profesional de los Docentes de Educación Infantil en su vinculación con la Educación Física (CDPEFI) (Losada, Honrubia, \& Gil, 2020). La aplicación del instrumento en futuros docentes de EI ofrecería la posibilidad de comparación con docentes en activo y analizar las necesidades en el proceso formativo en las universidades de cara a afrontar sus prácticas y su futura incorporación a la función docente.

\section{Conclusiones}

La investigación permitió describir la percepción de los estudiantes de último curso del grado de EI acerca de la situación de la motricidad en las aulas escolares. Se evidencia que los estudiantes otorgan una elevada importancia a la educación motriz y su trascendente inclusión en el currículo, no obstante, perciben que esta no se trabaja lo suficiente y es necesario un aumento de horas. Por otra parte, emanaron algunas evidencias respecto a las competencias del estudiante con las que afronta sus prácticas docentes en relación a la motricidad, que orientan el proceso formativo en la titulación universitaria.

\section{Referencias}

Abilleira, M. \& Fernández, M. (2017). Análisis de la formación del maestro/a de educación infantil en expresión corporal. EmásF: revista digital de educación física, 49, 36-59. Recuperado de https:/ /dialnet.unirioja.es/descarga/articulo/6195135.pdf

Aguado-Ruíz, F. (2014). Psicomotricidad: formación de los maestros de educación infantil y educación física. En P. Miralles-Martínez, M. ${ }^{\text {a B }}$ Alfageme-González \& R. A. Rodríguez Pérez(Eds.), Investigación e Innovación en Educación Infantil. (273-282). Murcia, España: Universidad de Murcia.
Alonso, Y. \& Pazos, J. M. (2020). Importancia percibida de la motricidad en Educación Infantil en los centros educativos de Vigo (España). Educação e Pesquisa, 46, 1-17.https://dx.doi.org/10.1590/s16784634202046207294

Arac'ilgar, E. \& Cihan, B. B. (2018). Metaphoric perceptions of school principals towards physical education term. International Journal of HigherEducation, 7(5), 194-205. https://doi.org/ 10.5430/ ijhe.v7n5p194

Berruezo, P. P. (2008). El contenido de la Psicomotricidad: reflexiones para la delimitación de su ámbito teórico y práctico. Revista Interuniversitaria de formación del profesorado 34, 25-32. Recuperado de https://dialnet.unirioja.es/descarga/articulo/2707331.pdf

Camina, P. (2013). Psicomotricidad y motricidad en educación infantil. (Tesis de grado). Universidad de Valladolid: Facultad de Educación y Trabajo Social. Recuperado de: https://uvadoc.uva.es/bitstream/ handle/10324/3958/TFG-G331.pdf

Campos, M. D. C., Lasaga, M. J. \& Ries, F. (2013). Evaluation of the training of early Chilhood Education teachers in Psychomotricity. In Ballesteros-Moscosio, M.A. \& Ries, F. (2013). International Conference Re-conceptualizing the professional identity of the European teacher, 801-814. Recuperado de https://idus.us.es/ bitstream/handle/11441/56718/RPIET_57.pdf

Castro, A. \& García-Ruiz, R. (2013). La visión del profesorado de Educación Infantil y Primaria de Cantabria sobre la participación y las relaciones interpersonales entre los miembros de la comunidad escolar. Aula Abierta, 41(1), 73-84. Recuperado de https:// dialnet.unirioja.es/descarga/articulo/4097758.pdf

Crespo, J. (2006). Descripción de la incidencia de las variables ambientales en los centros de educación infantil. Innovación Educativa, 16.299-310. Recuperado de https://minerva.usc.es/xmlui/bitstream/ handle/10347/4400/pg_299-310_inneduc16.pdf

Da Fonseca, V., Garrote, N., Todoli, D. \& Zenarruzabeitia, A. (2014). Los maestros analizan la importancia de la psicomotricidad en educación infantil. Madrid: Siena.

Díaz, R. \& Sosa, A. (2016). Percepción de los profesores sobre la importancia de la psicomotricidad en educación infantil. Acciónmotriz, 17, 7-20. Recuperado de https://dialnet.unirioja.es/ servlet/articulo?codigo $=6324706$

Dopico-Pedre, M. (2016). Estudio descriptivo sobre la formación inicial de los/as maestros y maestras de Educación Física escolar. Sportis Sci J, 2(2), 188 205. http://dx.doi.org/10.17979/ sportis.2016.2.2.1429

Espinosa, A., \& Vidanes, J. (1991). La nueva ordenación de la educación infantil. Madrid: Escuela española.

Fraile-Garciìa, J., Ibaibarriaga-Toset, A. \& Mon-Loìpez, D. (2019). Psicomotricidad en la etapa de infantil: situación actual en la enseñanza pública de la comunidad de Madrid. Revista Espanbola de Educacioìn Fiìsica y Deportes, 426 (Esp.), 270-280. Recuperado de http://www.reefd.es/index.php/reefd/article/viewFile/790/668

Gambau, V. (2015). Las problemáticas actuales de la educación física y el deporte escolar en España. Revista Española de Educación Física y Deportes, 411, 53-69. Recuperado de http://www.reefd.es/ index.php/reefd/article/view/114/111

García-Esteban, F. E. \& Murga-Menoyo, M. Á. (2015). El profesorado de educación infantil ante el desarrollo sostenible: necesidades formativas. Enseñanza \& Teaching, 33 (1), 121-142. http:// dx.doi.org/10.14201/et2014331121142

Gil-Espinosa, F., Romance, Á., \& Nielsen, A. (2018). Juego y actividad física como indicadores de calidad en Educación Infantil. Retos, 34, 252-257. Recuperado de https://recyt.fecyt.es/index.php/retos/ article/view/60391

Gil-Madrona, P., Contreras-Jordán, O. R., González-Víllora, S. \& Gómez-Barreto, I. M. (2008). Justificación de la Educación Física en la educación infantil. Educación y Educadores, 11(2), 159-177. https://doi.org/10.1174/021037008784132950

González, M. A., Sendón, S. R., Díaz, J. R., \& Gómez, C. A. (2018). Estudio descriptivo de las consideraciones sobre la importancia de 
la formación en expresión corporal de los futuros maestros y maestras de educación infantil. TRANCES. Transmisión del Conocimiento Educativo y de la Salud, 6(nov-dic), 739-758. Recuperado dehttps://dialnet.unirioja.es/servlet/articulo?codigo $=7076666$

González-Calvo, G. \& Martínez-Álvarez, L. (2018). Los Diarios Corporales Docentes como Instrumentos de Reflexión y de Evaluación Formativa en el Prácticum de Formación Inicial del Profesorado. Estudios pedagógicos (Valdivia), 44(2), 185-204. https:// dx.doi.org/10.4067/S0718-07052018000200185

Hernández, J.R., Cortegaza, L. \& Labrada, J.L. (2012). Lapsicomotricidad en la educación física contemporánea. CD de Monografías 2012, Universidad de Matanzas «Camilo Cienfuegos». Recuperado de https://docplayer.es/42911783-La-psicomotricidad-en-laeducacion-fisica-contemporanea.html

Hernández, R., Fernández, C. \& Baptista, P. (2003). Metodología de la investigación. México: Mc Graw-Hill Interamericana.

Latorre, P.A. \& López, J.M. (2009). Desarrollo de la motricidad en Educación Infantil: Consideraciones curriculares, científicas y didácticas. Granada: GEU.

Latorre, P.A. (2007). La motricidad en educación infantil, grado de desarrollo y compromiso docente. Revista iberoamericana de educación, 43(7), 1-7. https://doi.org/10.35362/rie4372296

Le Boulch, J.(1972). La educación por el movimiento en edad escolar. Buenos Aires: Paidós.

LOE. Ley Orgánica 2/2006, de 3 de mayo, de Educación(B.O.E. n 106, 4 de mayo de 2006).

Losada, L., Honrubia, C. \& Gil, P. (2020). Validación de un instrumento de desarrollo profesional en educación física infantil. Revista Internacional de Medicina y Ciencias de la Actividad Físicay del Deporte, 20(77), 57-72. Recuperado de https://revistas.uam.es/rimcafd/ article/view/rimcafd2020.77.004/11910

Madrona, P., Jordán, O. \& Barreto, I. (2008). Habilidades motrices en la infancia y su desarrollo desde una educación física animada. Revista iberoamericana de educación, 47(1), 71-96. Recuperado dehttps://dialnet.unirioja.es/descarga/articulo/2736759

Marinšek, M., Jurak, G. \& Kovaè, M. (2020). Differences in Beliefs Regarding Physical Education Between Slovenian In-Service and Pre-Service Early Childhood Educators. Journal of Research in Childhood Education, 34(2), 251-266. https://doi.org/10.1080/ 02568543.2019 .1676333

Mendoza, M. \& Covarrubias, C.G. (2014). Competencias Profesionales Movilizadas en el Prácticum: Percepciones del Estudiantado del Grado de Maestro en Educación Primaria. Actualidades Investigativas en Educación, 14(3), 204-229. Recuperado de https:/ /doi.org/10.15517/aie.v14i3.16089

Martínez-Bello, V., \& Bernabé-Villodre, M.M. (2019). Experiencia universitaria innovadora sobre didáctica de la motricidad infantil: De las aulas universitarias a las aulas de Educación Infantil. Revista Electrónica Educare, 23(3), 300-325. https://dx.doi.org/10.15359/ ree.23-3.15

Mérida-Serrano, R., Olivares-García, M. \& González-Alfaya, M. (2018). Descubrir el mundo con el cuerpo en la infancia. La importancia de los materiales en la psicomotricidad infantil. Retos, 34, 329-336. Recuperado de https://recyt.fecyt.es/index.php/retos/ article/view/64652/39887

Moreno, J. A., López, B., Gutiérrez, E. M., Cascada, M. \& Fernández, M. R. (2004). Situación actual de la motricidad en la etapa de 0 a 6 años según el profesorado de educación infantil. Revista iberoamericana de psicomotricidad y técnicas corporales, 16, 17-34. Recuperado de https://dialnet.unirioja.es/servlet/ articulo? codigo $=3739246$

Muradás, M. \& Porta, M.I. (2007). Las memorias del Prácticum I de maestros de educación infantil: sobre qué reflexionan los alumnos. En Alfonso Cid, María Muradás, Miguel Ángel Zabalza, Mercedes González, María Lina Iglesias y Manuela Raposo (Coord.), Buenas prácticas en el Prácticum (977-990). Santiago de
Compostela: Imprenta universitaria.

Pacheco, G. (2015). Psicomotricidad en Educación Inicial. Algunas consideraciones conceptuales. Ecuador: Primera Edición.

Palomero, J.E. (2007). La psicomotricidad en España en el contexto de la Convergencia Universitaria Europea. Revista iberoamericana de psicomotricidad y técnicas corporales, 7(1), 1-9. Recuperado de https://www.ugr.es/ recfpro/rev42COL2.pdf

Peña, V.J. \& Vicente, G. (2019). Danza en educación infantil: opinión de los docentes. Retos: nuevas tendencias en educación física, deporte y recreación, 36, 239-244. Recuperado dehttps://dialnet.unirioja.es/ descarga/articulo/7260909

Piaget, J.(1969). Biología y conocimiento: ensayo sobre las relaciones entre las regulaciones orgánicas y los procesos cognitivos. Madrid: Siglo XXI de España Editores.

Picq L. \& Vayer P. (1977). Educación psicomotriz y retraso mental. Barcelona: Científico-Médica.

Pons, R. \& Arufe, V. (2016). Análisis descriptivo de las sesiones e instalaciones de psicomotricidad en el aula de educación infantil. Sportis. Revista Técnico - Científica del deporte Escolar, Educación Física y Psicomotricidad, 2(1), 125 - 146. Recuperado de https://dialnet.unirioja.es/descarga/articulo/5294911

Quirós, V. (2000). Psicomotricidad y formación docente. Profesorado. Revista de currículum y formación del profesorado, 4(2), 1-9. Recuperado de $h$ ttp://goo.gl/xDQhy8

Rodríguez, P. F. \& Hernández, F. (2018). Didáctica de la Motricidad en la formación de profesores de educación infantil. Retos: nuevas tendencias en educación física, deporte y recreación, 34, 25-32. Recuperado de https://dialnet.unirioja.es/descarga/articulo/6736313

Ruiz, F., García,A., Gutiérrez, F., Marqués, J. L., Román, R. \& Samper, M. (2003). Los juegos en la motricidad infantil de los 3 a los 6 años. Editorial Inde.

Sáenz-López, P. (1999). La importancia de la Educación Física en Primaria. Revista Apunts, Ed. Física y Deportes, 57, 20-31. Recuperado de https://www.raco.cat/index.php/ApuntsEFD/article/view/ 306995/396974

Shingjergji, A. (2013). Psychomotor education, an aspect of general formation of pre-school children. The Journal of Education, Culture, and Society, 1, 121-132. https://doi.org/10.15503/jecs20131-121132

Simo, A. \& Espada, M. (2013). La intervención docente en psicomotricidad en los centros de educación infantil de Barcelona. Athlos: Revista internacional de ciencias sociales de la actividad física, el juego y el deporte, 5, 55-74. Recuperado de https:// dialnet.unirioja.es/descarga/articulo/5311417

Solís, A., Prieto, J. A., Nistal, P. \& Vázquez, M. M. (2017). Percepción y aplicación de la psicomotricidad por parte del profesorado de la etapa Infantil. Sportis, 3(1), 141-160. https://doi.org/10.17979/ sportis.2017.3.1.1794

Streiner, D.L. (2003). Starting at the beginning:an introduction to coefficient alpha and internal consistency. J Pers Assess, 80, 99103. https://doi.org/10.1207/S15327752JPA8001_18

Sugrañes-Justafré, E. \& Ángel-Ferrer, M. A. (2007). La educación psicomotriz (3-8 años): Cuerpo, movimiento, percepción, afectividad: Una propuesta teórica ( $1^{\mathrm{a}}$ ed.). Barcelona: Graó.

Torre, E. (2012). Significados de la Educación Física en el ámbito educativo. Un estudio desde las percepciones de los estudiantes de Magisterio. (Tesis doctoral). Granada: Universidad de Granada.

Vaca, M. J. (1988). Tratamiento pedagógico de lo corporal: el niño entero en el pensamiento del maestro. Tabanque: Revista pedagógica, 4, 147-158. Recuperado de https://dialnet.unirioja.es/descarga/articulo/2256966.pdf

Valdemoros, M., Sanz, E., Ponce de León, A. M. \& Alonso, R. A. (2018). Cualificación e implicación del profesorado de infantil frente a la educación motriz. Sportis, 4(1), 126-143. https://doi.org/ 10.17979/sportis.2018.4.1.3165 